\title{
Ferramenta de visualização georreferenciada para implantação da infraestrutura de rede móvel 5G no Brasil
}

\author{
Marcos Imperio $^{1}$, Gustavo Sousa Pavani ${ }^{1}$ \\ ${ }^{1}$ Universidade Federal do ABC (UFABC) \\ Av. dos Estados, 5001. Santo Andre - SP - Brasil. CEP: 09210-580. \\ \{m.imperio, gustavo.pavani\}@ufabc.edu.br
}

\begin{abstract}
The fifth generation mobile networks (5G) have already started to be deployed around the world. The implementation of such infrastructure in Brazil, whose frequency auction is scheduled for this year, is a great challenge when considering its continental dimensions. For reasons of investment reduction, it is assumed that such infrastructure will start from the current $4 G$ installed park. In this sense, the proposed visualization tool allows to see the location of $4 G$ base stations on the map of Brazil. It also makes it possible to view the future optical infrastructure, necessary to meet the requirements of $5 G$ connectivity, which is used to interconnect cities that already have $4 G$ coverage.
\end{abstract}

Resumo. As redes móveis de quinta geração (5G) já começaram a ser implantadas ao redor do mundo. A implantação de tal infraestrutura no Brasil, cuja leilão das frequências está previsto para este ano, é um grande desafio quando se considera suas dimensões continentais. Por motivos de redução de investimentos, pressupõe-se que tal infraestrutura partirá do parque instalado $4 G$ atual. Nesse sentido, a ferramenta de visualização proposta permite ver a localização das Estações Rádio Base (ERB) $4 G$ sobre o mapa do Brasil. Também possibilita visualizar a futura infraestrutura óptica, necessária para atender os requisitos de conectividade $5 G$, que é usada para interconexão das cidades que já possuem cobertura $4 G$.

\section{Introdução}

Em 2021, já está previsto o leilão de radiofrequências para a tecnologia móvel de 5G [ANATEL b], cujas regras já foram praticamente definidas pela Agência Nacional de Telecomunicações (ANATEL).

Estima-se que será necessário um grande investimento das operadoras móveis para a implantação das Estações Rádio Base (ERBs) na tecnologia 5G, além da infraestrutura de transporte em fibra óptica para atendimento dos requisitos do 5G [Mitra and Agrawal 2015, Jaber et al. 2016, Arévalo and Gaudino 2019].

Uma alternativa para redução desses investimentos é o compartilhamento de recursos entre operadoras com uso da infraestrutura 4G legada [Demirkol et al. 2017, Romero-Gázquez et al. 2019]. Nesse contexto, uma das formas que pode facilitar a compreensão desse problema é a visualização desse compartilhamento das ERBs e da infraestrutura óptica de transporte. 
Assim, o objetivo deste trabalho é propor uma ferramenta de visualização georreferenciada para as ERBs na tecnologia $4 \mathrm{G}$ junto com uma estimativa da infraestrutura óptica necessária para a implantação do 5G no Brasil.

Para atingir esse objetivo, são utilizados apenas dados abertos públicos que podem ser obtidos em:

- Sistema Integrado de Gestão e Controle do Espectro (MOSAICO) [ANATEL d] da ANATEL;

- Geolocalização e mapas do projeto Open Street Map (OSM) [OSM ].

O restante deste artigo é organizado da seguinte forma. Na Seção 2, são apresentados os dados que podem ser obtidos do sistema MOSAICO da ANATEL. Na Seção 3 é apresentada a arquitetura da ferramenta de visualização georreferenciada das ERBs 4G e da infraestrutura óptica. Na Seção 4, é mostrado o funcionamento da ferramenta. Por fim, na Seção 5, as conclusões deste artigo são apresentadas.

\section{Sistema Integrado de Gestão e Controle do Espectro (MOSAICO)}

Entre os tipos de dados oferecidos pelo sistema MOSAICO para cada ERB, obtidos neste trabalho em março de 2021, destacam-se:

1. O nome da operadora;

2. O identificador único da ERB;

3. O código IBGE do município da ERB [IBGE ];

4. O endereço da ERB;

5. As coordenadas geográficas da ERB, na forma de latitude e longitude (sistema SIRGAS2000 / WGS84 [ANATEL c]).

6. A frequência inicial e final de downlink para cada antena;

7. Tecnologia da antena (isto é, a geração - $2 \mathrm{G}, 3 \mathrm{G}$ ou $4 \mathrm{G}$ );

8. Classificação da infraestrutura física (isto é, Greenfield ${ }^{1}$ ou Rooftop ${ }^{2}$ ); e

9. Se há algum tipo de compartilhamento da infraestrutura física.

Entretanto, as duas últimas informações estão listadas de forma incompleta ou não padronizada. Assim, para efeitos de compartilhamento de frequência, se considera que se uma operadora faz uso de uma subfaixa de frequência licenciada para outra operadora, há compartilhamento de espectro por essa operadora.

As atuais subfaixas de frequência regulamentadas pela ANATEL estão mostradas na Tabela 1 do Anexo, considerando-se o downlink, isto é, as frequências utilizadas na comunicação da ERB para o celular.

Além disso, para se efetuar a visualização e o respectivo cálculo das distâncias, é preciso utilizar a conversão de coordenadas em decimais, pois os valores públicos de latitude e longitude obtidos pelo sistema MOSAICO encontram-se com a notação em minutos e segundos, além de ter caracteres indicando sul, norte e oeste.

Vale a pena ressaltar que há casos em que as localizações geográficas e/ou endereços de algumas estações não estavam condizentes com o estado ou cidade. Nesse caso, foi necessária a verificação e correção manual desses casos identificados.

\footnotetext{
${ }^{1}$ Instaladas no solo.

${ }^{2}$ Instaladas na cobertura de edifícios.
} 
Mais ainda, há imprecisão nos dados de geolocalização das ERBs presentes no sistema MOSAICO. Dessa forma, foi necessário determinar um raio máximo para considerar que duas ou mais ERBs distintas podem compartilhar a infraestrutura entre si. Nesse trabalho, considerou-se um raio de 50 metros, que se mostrou acertado para uma série de testes realizados, em que se fez uso de banco de imagens públicos de imagens obtidas via satélite.

\section{Ferramenta de visualização web georreferenciada}

A ferramenta de visualização web permite localizar as ERBs $4 \mathrm{G}$ no Brasil de forma interativa, além de estimar a infraestrutura óptica de interligação das localidades atendidas.

Os dados das ERBs $4 \mathrm{G}$ podem ser obtidos diretamente do sistema MOSAICO, como descrito na Seção 2.

Os banco de dados de ERB foi exportado em JavaScript Object Notation (JSON), conforme mostrado abaixo, criando-se um arquivo para cada estado:

- Operadora móvel (operadora);

- Identificador único da ERB (id);

- Código IBGE do município (codMunicipio) [IBGE ];

- Coordenadas geográficas (lat e long);

- Indicação de compartilhamento de espectro com outra operadora (spectrum).

Entretanto, ao contrário do que acontece na telefonia móvel, não há dados abertos fornecidos pela ANATEL para a infraestrutura óptica no Brasil. Assim, é necessária alguma forma de estimar a topologia dessa infraestrutura.

Para tanto, considera-se que as cidades que pertencem a um prefixo de DDD estariam agrupadas em um anel de fibra óptica, que tem como vantagens a sobrevivência e a eficiência energética [Wong et al. 2017], constituindo o backhaul para determinada operadora móvel ou para a rede neutra [ANATEL a], que seria a rede óptica compartilhada e passível de contratação por qualquer operadora móvel.

O cálculo das rotas envolvidas na construção do backhaul de cada DDD é efetuado de forma off-line pelo software OptaPlanner [OptaPlanner ]. O OptaPlanner é um software de código aberto (open source) para a solução de problemas com restrições, como é o caso do problema do caixeiro viajante (TSP) [Applegate et al. 2006] deste trabalho.

O problema do caixeiro viajante pode ser definido da seguinte forma: dado um conjunto de cidades a serem visitadas e o custo da viagem entre cada par de cidades, qual seria a rota mais curta para visitar todas as cidades, retornando a origem? Dessa forma, com uma boa solução para o problema do caixeiro viajante, minimiza-se a distância a ser coberta pelo anel de fibra óptica.

Assim, considera-se que a primeira ERB de tecnologia $4 \mathrm{G}$ de cada cidade é selecionada para fazer parte do percurso a ser otimizado no problema do caixeiro viajante.

As distâncias geográficas das ERBs selecionadas são obtidas da base de dados do OpenStreetMap com o uso do software GraphHopper [GraphHopper ]. Quando há algum eventual erro no cálculo de distância, o que é pouco comum, se utiliza no lugar o valor da distância de Haversine, que fornece a distância entre dois pontos de uma esfera, multiplicada por 1,3 para se estimar melhor os eventuais desvios da rota geográfica 
[Chiu et al. 2009]. É importante ressaltar que, em caso de erro do cálculo de distância, não é possível visualizar o backhaul na ferramenta.

Para cada estado e para cada operadora/rede neutra, é exportado o arquivo do backaul resultante no formato JSON, descrevendo um conjunto de paradas (waypoints) por DDD. Note que esse arquivo descreve as coordenadas das ERBs na ordem determinada pela solução problema do caixeiro viajante.

O fluxograma da Figura 1 apresenta uma forma resumida da interação e sequência utilizada para geração de arquivos em JSON, que posteriormente são utilizados na ferramenta de visualização web.

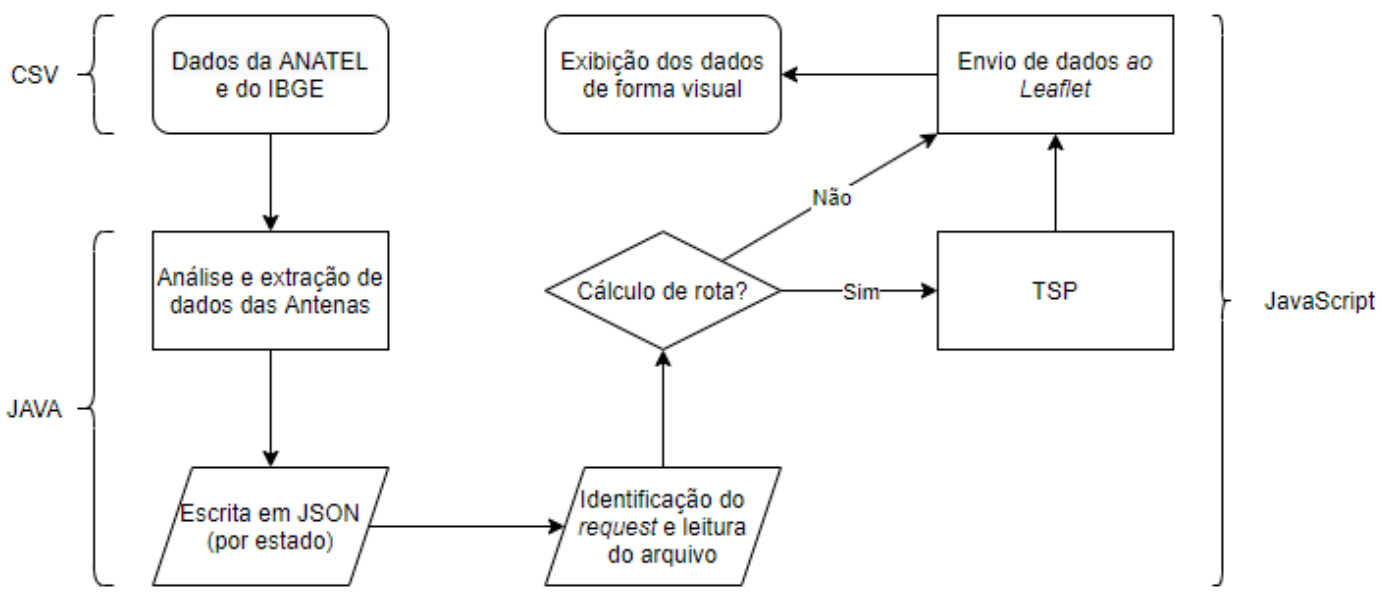

Figura 1. Fluxograma de processamento dos dados públicos utilizados pela ferramenta web.

Para a implementação da ferramenta de forma online, foi utilizado:

- Software de mapa: Open Street Map (OSM) [OSM ], que constitui um mapa editável aberto do mundo que está sendo continuamente construído e melhorado por voluntários. Sua licença, que é do tipo Open Database License, permite o acesso gratuito das imagens e dos dados relacionados. Para este trabalho, foi utilizada a versão obtida na data de 16 de março de 2020;

- Biblioteca para projeção no mapa: Leaflet [Leaflet ], que é predominante escrita na linguagem JavaScript. É uma biblioteca de código aberto bastante utilizada e que permite a visualização de mapas interativos;

- Linguagens de programação: predominantemente HTML, CSS e JavaScript;

- Cálculo de rotas: software Open Source Routing Machine (OSRM) [OSRM ] instalado localmente. Representa um motor de roteamento de código aberto escrito em $\mathrm{C}++$ e que é utilizado pelo Leaflet.

\section{Funcionamento da ferramenta}

A ferramenta possui as seguintes funcionalidades para auxiliar o usuário:

- Indicação das principais informações relativas a cada ERB, como identificador, latitude, longitude e compartilhamento de espectro;

- Indicação de rota para interconexão entre duas ERB; 
- Raio de cobertura de uma ERB;

- Visualização da topologia do backhaul óptico.

A ferramenta leva em consideração as oito operadoras de redes móveis celulares em operação no Brasil, sendo que cada operadora móvel recebeu uma legenda diferente, inspirada nas cores dos respectivos logotipos, conforme Figura 2, na qual se visualiza a cidade de Rio Branco, Acre.

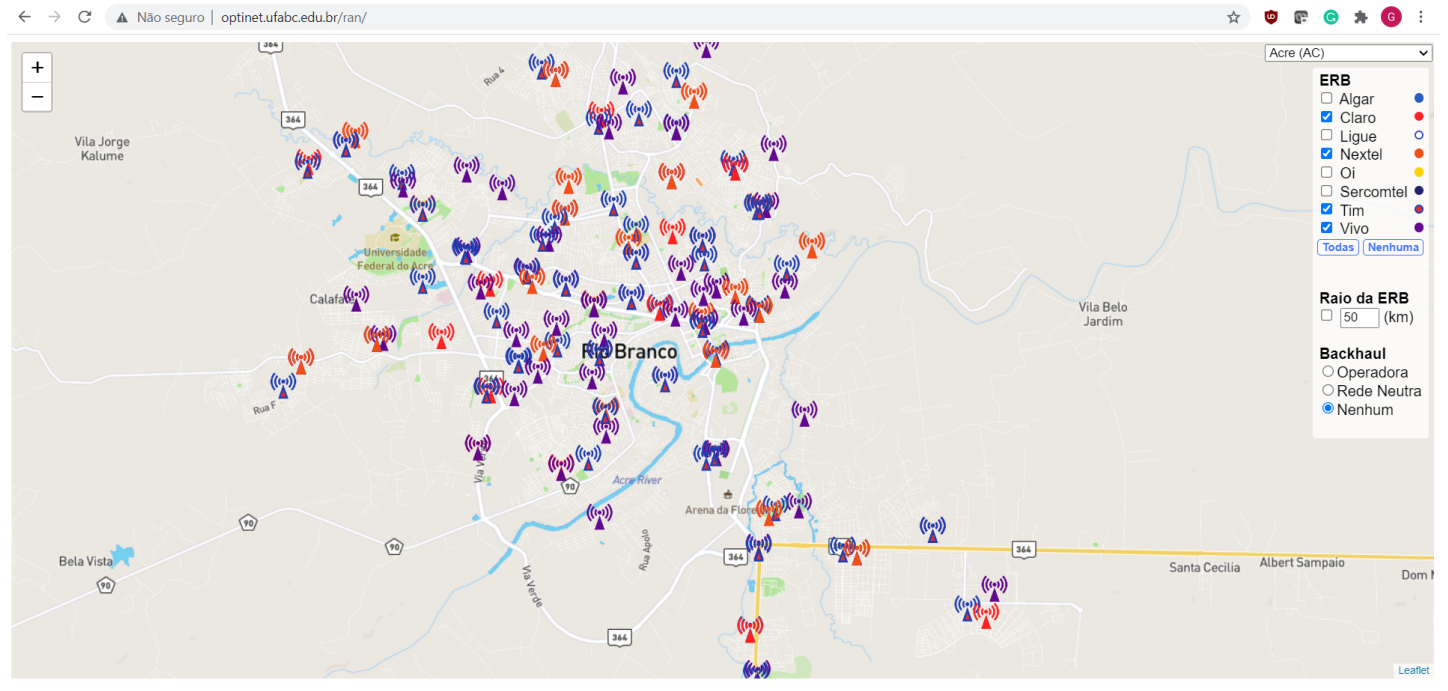

Figura 2. Visualização das ERBs em Rio Branco, Acre.

Note que, dada a grande quantidade de dados envolvidos, é possível selecionar para cada estado brasileiro quais as operadoras móveis que serão mostradas na tela.

A Figura 3 ilustra o uso da ferramenta para o estado do Acre, com uma rota sendo traçada entre duas ERBs. É possível ver ainda as informações relativas a ERB selecionada, sendo que há compartilhamento de espectro com as operadoras Vivo e Oi.

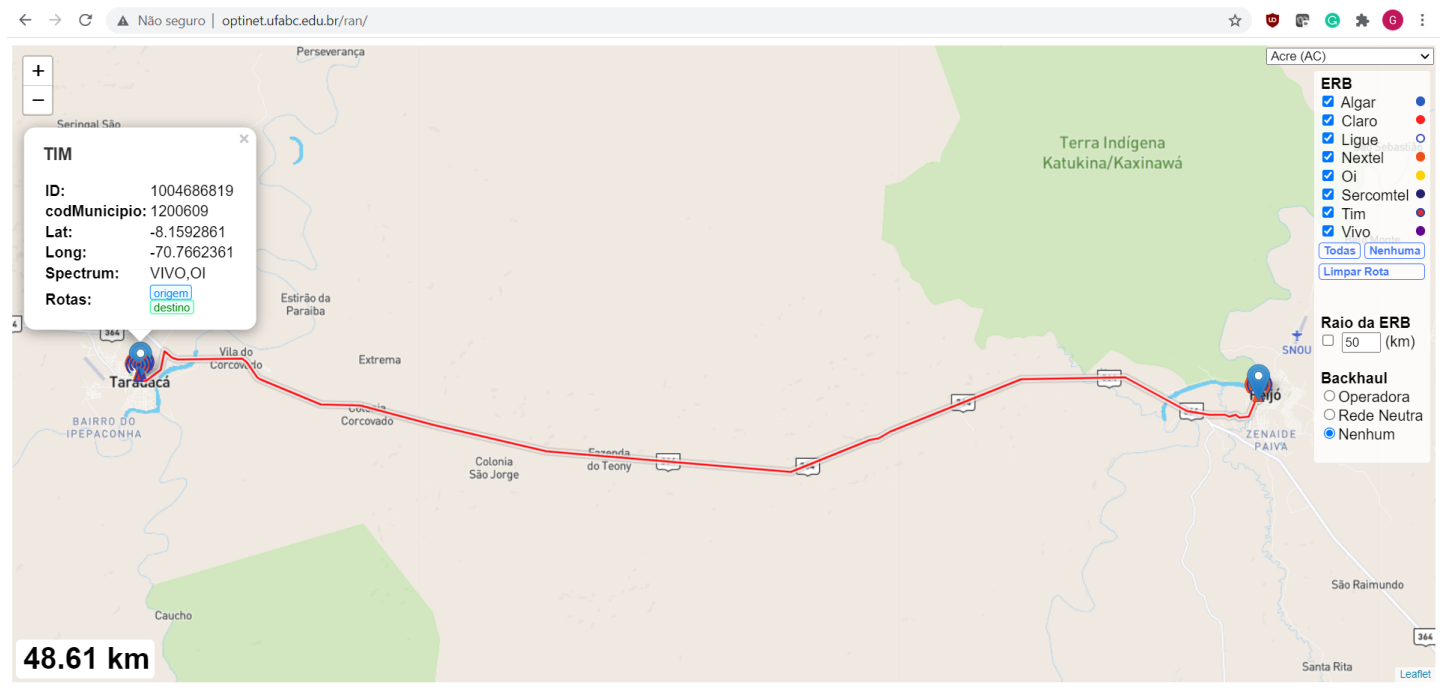

Figura 3. Informações sobre as ERBs e visualização de rota.

Também há possibilidade de inserir o raio de cobertura de cada estação (em quilômetros), conforme mostrado na Figura 4. 


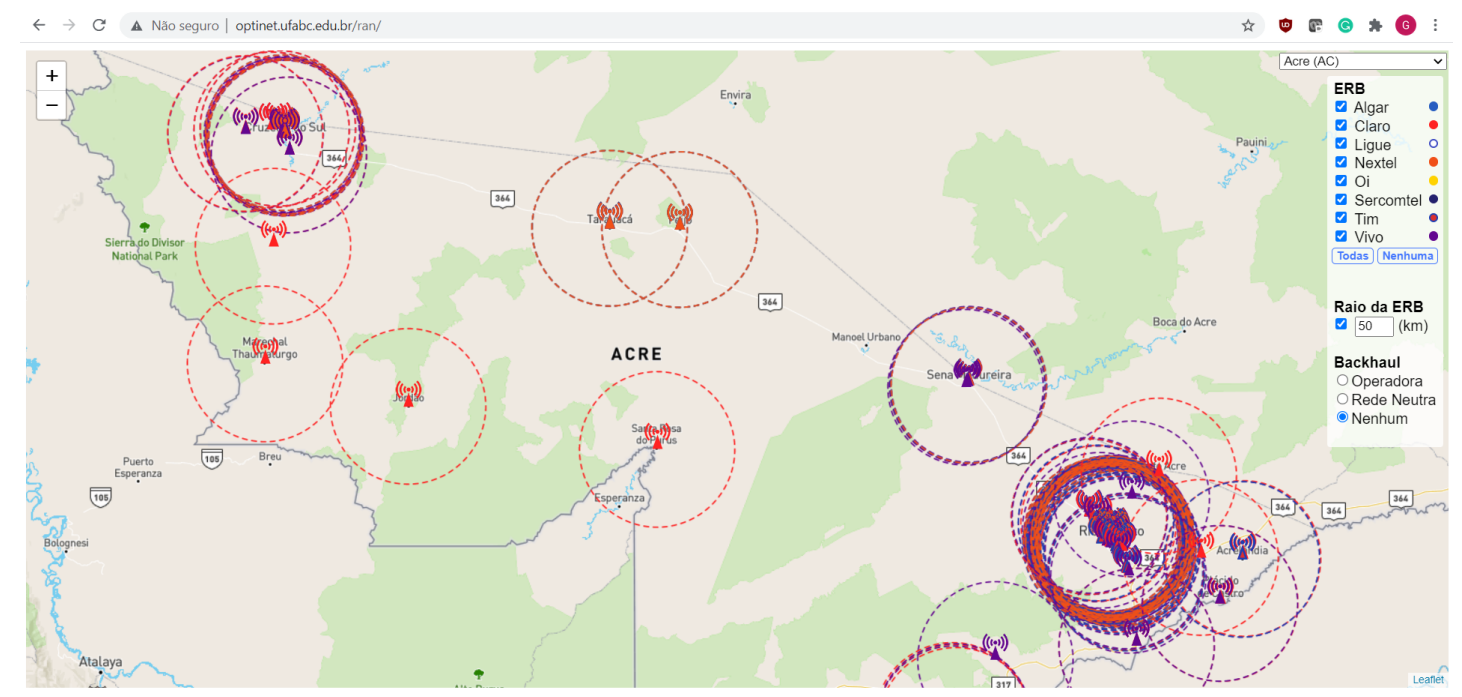

Figura 4. Visualização de um raio de cobertura por ERB de $50 \mathrm{~km}$ no Acre.

Por fim, outra funcionalidade criada é visualizar o backhaul óptico para uma operadora ou da rede neutra. A Figura 5 apresenta o backhaul óptico da rede da operadora TIM no estado do Acre, que compõe o DDD 68.

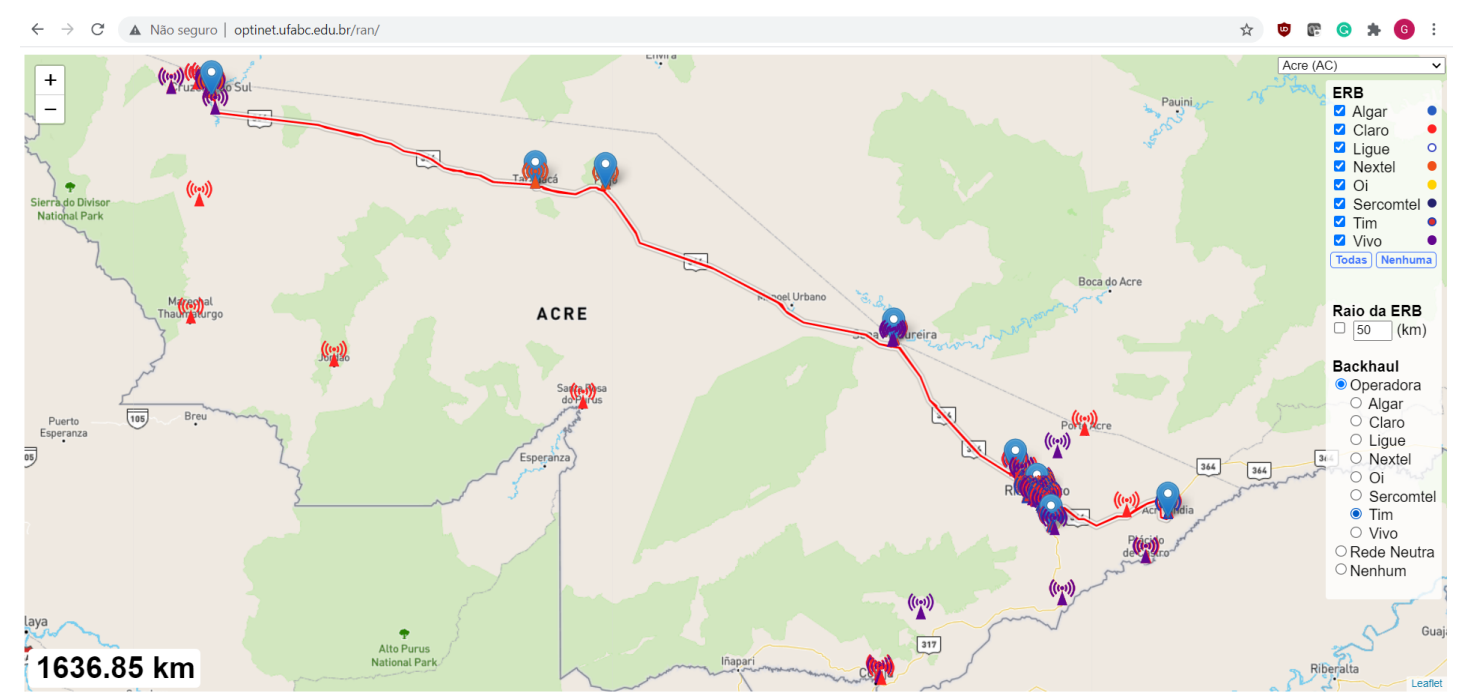

Figura 5. Visualização do backhaul óptico da rede da operadora TIM para o Acre.

Observe que, devido às poucas estradas disponíveis, há compartilhamento de estradas nos enlaces de fibras ópticas de tal forma que grande parte do anel não é disjunto do ponto de vista dos caminhos.

\subsection{Maiores informações}

A ferramenta está disponível em: http://optinet.ufabc.edu.br/ran

A respectiva documentação pode ser encontrada em: http://optinet. ufabc.edu.br/ran/manual

Por fim, foi disponibilizado o seguinte vídeo explicando as funcionalidades da ferramenta: https: / / www . youtube. com/watch?v=3 JcA8T3Py2I. 


\section{Conclusão}

Neste artigo foi apresentada a ferramenta georreferenciada que visa auxiliar a implantação do $5 \mathrm{G}$ no Brasil.

Com efeito, ela permite visualizar a rede legada de ERBs 4G, além do backhaul óptico necessário para a futura interconexão das cidades. Dessa forma, acredita-se que ela possa auxiliar nas decisões de implantação da infraestrutura necessária para alcançar tal objetivo.

\section{Agradecimentos}

Os autores gostariam de agradecer o apoio financeiro concedido por meio do processo no 2015/24341-7, Fundação de Amparo a Pesquisa do Estado de São Paulo (FAPESP).

\section{Referências}

ANATEL. Consulta Pública no 65/2020. https: / / sistemas.anatel.gov.br/ SACP / Contribuicoes/TextoConsulta.asp?CodProcesso=C2378\& Tipo=1\&Opcao=andamento.

ANATEL. Leilão de Espectro 5G. https://www.gov.br/anatel/pt-br/ assuntos/5G/leilao-de-espectro-5g. Acessado em 20 de maio de 2021.

ANATEL. Resolução no 571, de 28 de setembro de 2011. https: / / www . anatel. gov.br/legislacao/resolucoes/26-2011/44-resolucao-571.

ANATEL. Sistema Integrado de Gestão e Controle do Espectro (MOSAICO). http : / / sistemas.anatel.gov.br/se/public/view/b/licenciamento.php - Acessado em 5 de março de 2021.

Applegate, D. L., Bixby, R. E., Chvatál, V., and Cook, W. J. (2006). The Traveling Salesman Problem: A Computational Study. Princeton University Press.

Arévalo, G. V. and Gaudino, R. (2019). Optimal dimensioning of the 5G optical fronthaulings for providing ultra-high bit rates in small-cell, micro-cell and femto-cell deployments. In 21st International Conference on Transparent Optical Networks (ICTON), pages $1-4$.

Chiu, A. L., Choudhury, G., Clapp, G., Doverspike, R., Gannett, J. W., Klincewicz, J. G., Li, G., Skoog, R. A., Strand, J., Lehmen, A. V., and Xu, D. (2009). Network design and architectures for highly dynamic next-generation IP-over-optical long distance networks. Journal of Lightwave Technology, 27(12):1878-1890.

Demirkol, I., Camps-Mur, D., Bartelt, J., and Jim Zou (2017). 5G transport network blueprint and dimensioning for a dense urban scenario. In European Conference on Networks and Communications (EuCNC), pages 1-6.

GraphHopper. The GraphHopper Directions API Route Planning For Your Application. https://www.graphhopper.com/.

IBGE. Códigos dos municípios. https://www.ibge.gov.br/explica/ codigos-dos-municipios.php. [Acessado em 20-Setembro-2020, às 17:00].

Jaber, M., Imran, M. A., Tafazolli, R., and Tukmanov, A. (2016). 5G backhaul challenges and emerging research directions: A survey. IEEE Access, 4:1743-1766. 
Leaflet. An open-source JavaScript library for mobile-friendly interactive maps. https: //leafletjs.com/.

Mitra, R. N. and Agrawal, D. P. (2015). 5G mobile technology: A survey. ICT Express, 1(3):132-137. Special Issue on Next Generation (5G/6G) Mobile Communications.

OptaPlanner. AI constraint solver. https: / /www. optaplanner.org.

OSM. Open Street Map. https://wiki.openstreetmap.org/.

OSRM. Open Source Routing Machine. http://project-osrm.org/.

Romero-Gázquez, J., Moreno-Muro, F., Garrich, M., Bueno-Delgado, M., Khodashenas, P. S., and Pavón-Mariño, P. (2019). A use case of shared 5G backhaul segment planning in an urban area. In 21st International Conference on Transparent Optical Networks (ICTON), pages $1-4$.

Wong, E., Grigoreva, E., Wosinska, L., and Machuca, C. M. (2017). Enhancing the survivability and power savings of $5 \mathrm{G}$ transport networks based on DWDM rings. $J$. Opt. Commun. Netw., 9(9):D74-D85.

\section{Anexo}

Tabela 1. Subfaixas de frequência regulamentadas no Brasil (MHz).

\begin{tabular}{ll}
\hline Subfaixa & ERB para celular $($ Downlink $)$ \\
\hline A & $869 \vdash 880 / 890 \vdash 891,5$ \\
B & $880-890 / 891,5 \vdash 894$ \\
D & $1805 \vdash 1820$ \\
D' & $955 \vdash 957,5$ \\
E & $1835 \vdash 1850$ \\
E & $957,5 \vdash 960$ \\
F & $2110 \vdash 2125$ \\
G & $2125 \vdash 2135$ \\
H & $1885 \vdash 1890 / 1890 \vdash 1895 / 2135 \vdash 2140 / 2140 \vdash 2145$ \\
I & $2145 \vdash 2155$ \\
J & $2155 \vdash 2165$ \\
L & $1975 \vdash 1980 / 2165 \vdash 2170$ \\
M & $1850 \vdash 1860 / 1860 \vdash 1865 / 1865 \vdash 1870$ \\
P & $2620 \vdash 2630 / 2500 \vdash 2510$ \\
S & $461 \vdash 468 / 763 \vdash 803$ \\
W & $2510 \vdash 2530 / 2630 \vdash 2650$ \\
V1 & $2650 \vdash 2660$ \\
V2 & $2660 \vdash 2670$ \\
X & $2670 \vdash 2690$ \\
APT & $763 \vdash 803$ \\
Extensão 2G & $943,5 \vdash 946 / 952,5 \vdash 955$ \\
Extensão 3G & $1820 \vdash 1835 / 1860 \vdash 1870 / 1870 \vdash 1880 / 1885 \vdash 1895$ \\
\hline
\end{tabular}

